\title{
DESIGN OF A 35 keV LEBT FOR THE NEW HIGH INTENSITY OPPIS AT BNL
}

\author{
J. Alessi, M. Okamura", D. Raparia, T. Roser, BNL, NY, U. S. A. \\ C. D. P. Levy, A. Zelenski ${ }^{+}$, TRIUMF, Vancouver, Canada \\ T. Takeuchi, Konan Univ., Kobe, Japan \\ Y. Mori, KEK, Tsukuba, Japan
}

\begin{abstract}
As a part of the RHIC Spin project, the KEK optically pumped polarized ion source (OPPIS) is being upgraded at TRIUMF, and will then be installed at BNL. This new source will deliver $>100$ times more current than the existing BNL polarized $\mathrm{H}^{-}$source. In order to transport and accelerate this more intense polarized beam efficiently, a new $35 \mathrm{keV}$ low energy beam transport (LEBT) line has been designed. Each beamline element has been designed to minimize aberrations using 2D and 3D-field analysis. Spin motion and beam optics were tracked from the ionizer cell in the source to the $200 \mathrm{MHz}$ RFQ, including 3D-field effects.
\end{abstract}

\section{INTRODUCTION}

The KEK OPPIS is being upgraded at TRIUMF, and has now produced $1.0 \mathrm{~mA}$ of $\mathrm{H}^{-}$beam with $\mathrm{DC}$ mode. Also duration of a pumping laser has reached $200 \mu \mathrm{s}$ [1 - 3]. At $\mathrm{BNL}$, a new beamline was designed to match from this source into the $200 \mathrm{MeV}$ linac. The expected beam emittance from this OPPIS is much larger than that of existing atomic type polarized source, and the beam should be matched to the acceptance of the existing RFQ without depolarization. Therefore, this beamline must be designed carefully.

\section{SCHEME OF THE LEBT}

The normalized beam emittance determined empirically from similar OPPIS is about $2 \pi \mathrm{mm}$ mrad, while a calculation discussed later in this paper predicts a value of $2.35 \pi \mathrm{mm} \mathrm{mrad}(100 \%)$. This is approximately ten times of the emittance from the existing atomic beam polarized $\mathrm{H}^{-}$source now being used for AGS, and also larger than the emittance of the $\approx 100 \mathrm{~mA}$ unpolarized $\mathrm{H}^{-}$beam. The beam from the new source will be merged to the existing high intensity beam line and injected into the RFQ, which has an acceptance which is almost the same as the emittance from the OPPIS.

When designing the LEBT for a polarized beam, not only the beam optics but also spin motion must be considered. Beam leaving the OPPIS has longitudinal polarization, and should be rotated to the vertical direction before

\footnotetext{
\# Visitor from RIKEN okamura@bnl.gov
}

${ }^{+}$Visitor from INR

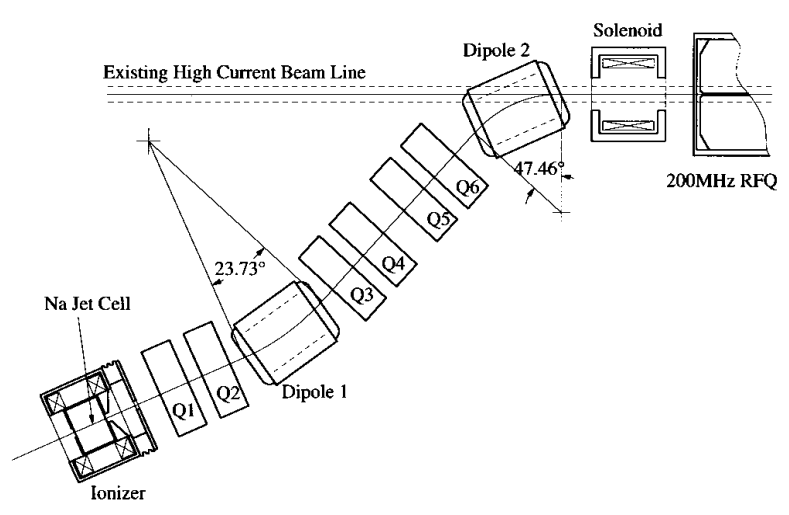

Figure 1: The LEBT for the new OPPIS

injection into the booster synchrotron. Figure 1 shows the planned beam line before the RFQ. First the beam from the source is accelerated to $35 \mathrm{keV}$ in the extraction gap, goes through a quadrupole doublet, and is deflected by 23.73 degrees in Dipole 1 . In this dipole, the spin direction is rotated by $90^{\circ}$, making it perpendicular to the beam direction and in horizontal plane. The beam then goes through two more quadrupole doublets and is then merged to the existing beamline by using $47.46^{\circ}$ bending magnet. This dipole will be pulsed, to allow switching between polarized and unpolarized $\mathrm{H}^{-}$beam on a pulse-topulse basis. In this dipole the spin direction is rotated by $180^{\circ}$, so is still perpendicular to the beam direction and in the horizontal plane. After this, the beam is focused strongly by a pulsed solenoid magnet and injected into the RFQ. At the same time, the spin rotates more than one revolution in the transverse plane. The exact rotation is not determined because the field strength of the solenoid will be adjusted to maximize transmission through the RFQ. After acceleration from $35 \mathrm{keV}$ to $750 \mathrm{keV}$, the beam then goes through another pulsed solenoid, added to $750 \mathrm{keV}$ beam, and there are sufficient quadrupoles existing in this line to allow flexibility in matching to

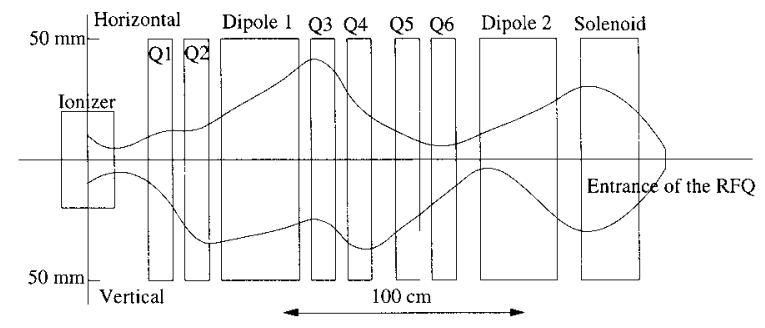

Figure 2: The beam envelope at the LEBT 
the $200 \mathrm{MeV}$ allow one to rotate the spin to the vertical direction. This solenoid has minimal effect on the transverse optics of the Alvarez linac. The beam envelope for the $35 \mathrm{keV}$ line, calculated using TRANSPORT, is shown in Fig. 2.

\section{BEAM EMITTANCE FROM THE OPPIS}

To start designing the LEBT, the beam emittance of the OPPIS had to be investigated. Figure 3 shows a schematic of the OPPIS.



Figure 3: Schematic of OPPIS

The OPPIS consists of two solenoid magnets, a $2.5 \mathrm{~T}$ super-conducting magnet, which includes an ECR cavity and $\mathrm{Rb}$ vapor cell, and a $1.4 \mathrm{kGauss}$ ionizer solenoid that has the $\mathrm{Na}$ vapor cell. $\mathrm{H}^{+}$ions are produced in the ECR cavity and extracted at $3.5 \mathrm{keV}$. The protons pick up a polarized electron in the $\mathrm{Rb}$ cell, to become neutral. The neutralized $\mathrm{H}$ beam drifts without effect from the magnetic fields to the $\mathrm{Na}$ cell, where it picks up an electron to become $\mathrm{H}^{-}$. The $\mathrm{H}^{-}$beam then leaves the OPPIS under the influence of fringing field of ionizer solenoid. The initial emittance of the OPPIS depends on where the neutralized beam becomes $\mathrm{H}^{-}$, and how the fringing field of the solenoid affects the $\mathrm{H}^{-}$beam. The emittance has been calculated using Monte Carlo for the charge exchange process, and Runge Kutta for particle tracking through electric and magnetic field maps created using OPERA2D. Spin motion was calculated by the Runge Kutta method, and the effect of the fringing field of the ionizer was analyzed. In this new OPPIS, a jet type $\mathrm{Na}$ cell, which has been recently developed at TRIUMF, will be used. We hope that with this new $\mathrm{Na}$ cell, the extraction voltage, accelerating from $3.5 \mathrm{keV}$ to $35 \mathrm{keV}$, can be achieved by biasing only the ionizer at high voltage, due to much less flow of $\mathrm{Na}$ vapor to outside. The electric field of the beam extraction was optimized to produce a waist in the beam at the center of the fringing region of the solenoid, so the effect of transverse magnetic fields to the spin motion can be minimized. As a result, calculations predict that depolarization due to the fringe field will be reduced by about 3\%. Figure 4 and Fig. 5 show the electric and magnetic fields calculated by OPERA-2D and the projection of the $\mathrm{H}$ particles respectively. The distribution of $\mathrm{Na}$ vapor was assumed to be Gaussian and $\sigma$ was set to $20 \mathrm{~mm}$. The total gas thickness was assumed to be $1.5 \times 10^{15}$ atoms $/ \mathrm{cm}^{2}$. Only two process were considered, $\mathrm{H}^{0} \rightarrow \mathrm{H}^{-}$and $\mathrm{H}^{-} \rightarrow \mathrm{H}^{0}$, as charge exchange process between $\mathrm{H}$ and $\mathrm{Na}$ atoms, with cross sections of

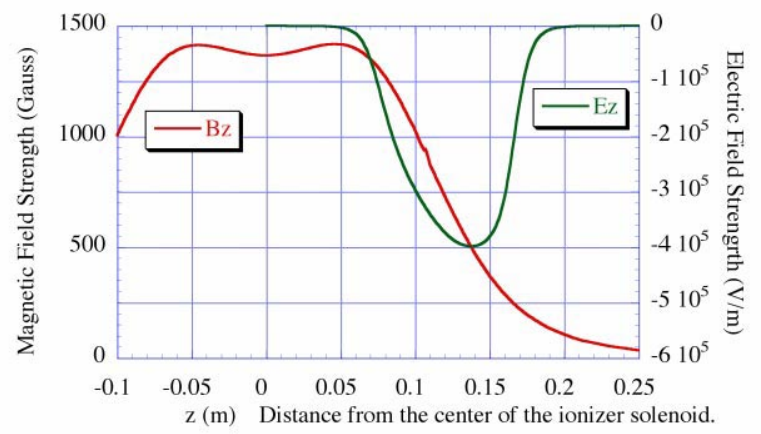

Figure 4: Field distribution of the Ionizer solenoid.

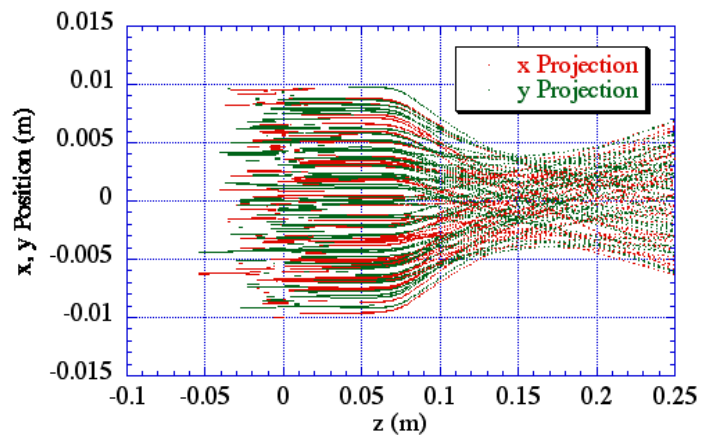

Figure 5: Projection of $\mathrm{H}^{-}$particles

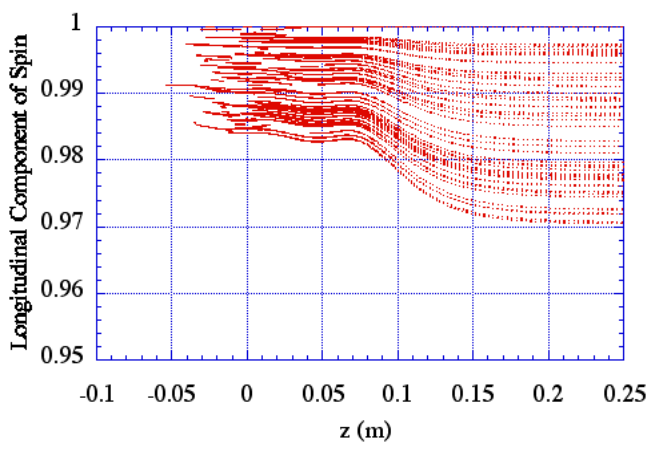

Figure 6: Spin motion at the ionizer cell

$0.03 \times 10^{-14} \mathrm{~cm}^{2}$ and $0.30 \times 10^{-14} \mathrm{~cm}^{2}$ respectively. At this beam energy, other charge exchange cross sections are negligible. Five thousand neutral protons were created uniformly within a circle of $10 \mathrm{~mm}$ radius and $1.0 \mathrm{mrad}$ of divergence, and were tracked from $20 \mathrm{~cm}$ upstream of the center of the solenoid. The yield of $\mathrm{H}^{-}$ions was $9.0 \%$. The predicted beam emittance $35 \mathrm{~cm}$ downstream from the center of the solenoid are given in Table 1. The calculated spin motion is shown in Fig. 6. Assuming $100 \%$ initial longitudinal polarization of the beam, a polarization of 
98.49\% was calculated after passing through the fringing field.

Table 1: Twiss parameters of the beam from theOPPIS

\begin{tabular}{|c|c|}
\hline$\varepsilon(100 \%)$ & $270.4 \mathrm{~mm} \mathrm{mrad}$ \\
\hline$\alpha$ & 3.81 \\
\hline$\beta$ & $0.903 \mathrm{~mm} / \mathrm{mrad}$ \\
\hline$\gamma$ & $17.2 \mathrm{mrad} / \mathrm{mm}$ \\
\hline
\end{tabular}

\section{COMPONENTS OF THE LEBT}

Each device in the beamline was designed to minimize spin aberrations. The six quadrupoles in this line will be electrostatic. In this section, only the dipole and solenoid magnets are discussed. Again, the Runge Kutta method was used in particle tracking with field maps created using OPERA-2D and 3D (TOSCA).

\subsection{Dipole Magnets}

To maintain the existing high current beam line, Dipole 2 $\left(47.46^{\circ}\right)$, shown in Fig. 2, has to have at least $10 \mathrm{~cm}$ of aperture and be less than $30 \mathrm{~cm}$ in length. The bending angle of Dipole 1 is only $23.73^{\circ}$, but the identical design



Figure 7: Over view of the Dipole magnet

Table 2: Effect of dipole magnets

\begin{tabular}{|l|c|c|}
\hline \multicolumn{1}{|c|}{ Magnet } & Dipole 1 & Dipole 2 \\
\hline Hor. emit. growth (rms) & $7.9 \%$ & $6.7 \%$ \\
\hline Ver. emit. growth (rms) & $2.4 \%$ & $4.2 \%$ \\
\hline Conserved spin & $99.96 \%$ & $99.82 \%$ \\
\hline
\end{tabular}

as Dipole 2 was applied due to large divergence from the OPPIS. In order to minimize spin aberrations, a rectangular shape was adopted so each particle sees almost the same integral of magnetic field, so has almost the same spin rotation. There is strong edge focussing of the beam in the vertical direction. An overview of the dipole design is shown in Fig. 7. The calculated emittance growth, using rms emittance, and conserved spin component are shown in Table 2.

\subsection{Solenoid}

The solenoid lens is the best focusing element for matching of the high current $(100 \mathrm{~mA}) \mathrm{H}^{-}$beam into the RFQ. However, we must pay careful attention to the spin motion for the polarized beam, since the spin axis rotates around the magnetic axis of the solenoid. As mentioned above, the spin rotation angle will vary due to fine adjustment of the solenoid field strength for focusing. Therefore, there will be another solenoid after the RFQ to align the spin direction vertically. Figures 8 shows the spin motion of an on-axis particle. The calculated remaining spin component is $99.6 \%$. The aperture and length of the solenoid magnet are $108 \mathrm{~mm}$ and $241.3 \mathrm{~mm}$ respectively, and the calculated effective length is 205.8 $\mathrm{mm}$. This magnet will be operated at $\approx 3.3 \mathrm{kGauss}$ at the center of the magnet to match to the acceptance of the RFQ.



Figure 8: Spin motion in the solenoid magnet.

\section{CONCLUSION}

The LEBT for the new BNL OPPIS was designed considering 3D field effects (except quadrupoles), and spin depolarization. The expected total conserved polarization is calculated to be better than $97.8 \%$ from the source ionizer to injection into the RFQ. The emittance growths for both planes through the line are less than $28 \%$, and the predicted RFQ transmission is $70 \%$.

\section{REFERENCES}

[1] M. Kinsho, et al., "Production of Polarized Negative Deuterium Ion Beam with Dual Optical Pumping" Review of Scientific Instruments, Vol. 67-3, 1362-1364 (1996)

[2] A. N. Zelenski, et al., "Optically Pumped Polarized $\mathrm{H}^{-}$Ion Sources for RHIC and HERA Colliders" These Proceedings.

[3] C. D. P. Levy, et al., "Development of High-Current Polarized H" Ion Souces at TRIUMF", EPAC'98, TUO028B, Stockholm, (1998) 